\section{Maturação, composição corporal e aptidão cardiorrespiratória em crianças e adolescentes na área do grande Porto, Portugal}

\section{Maturation, body composition and cardiorespiratory fitness in children and adolescents in the metropolitan area of Porto, Portugal}

Carol Leandro 1

Sandra Guerra 2

José Alberto Duarte 3

Jorge Mota 4

1,2,4 Gabinete de Desporto de Recreação e Tempos Livres, Faculdade de Ciências do Desporto e de Educação Física do Porto, Portugal. Rua Dr. Plácido Costa, 91. Porto, Portugal. CEP: 4200450

3 Laboratório de Morfologia Experimental da Faculdade de Ciências do Desporto e de Educação Física da Universidade do Porto, Portugal.

\begin{abstract}
Objectives: to determine in Portuguese students ( $n=256$ boys and $n=268$ girls) between eight and 16 years old: the patterns of changes in body composition and $\mathrm{VO}_{2 \max }$ related to the maturation process; the relationship between fat mass percentage (\%FM), body mass index (BMI), the sum of skin folds ( $5 S K F)$ and $\mathrm{VO}_{2 \max }$; the percentage of obese students.

Methods: body fatness was estimated from $\mathrm{SSKF}$ (subscapular and tricipital), and the distribution pattern obtained from the ratio between subscapular and tricipital skin fold thickness. Children were subdivided into maturation stages according to Tanner's staging for sexual maturity. $V O_{2 \max }$ was assessed by maximal multistage 20-meters Shuttle-run Test.

Results: anthropometric variables and fat mass percentage were consistent with earlier published studies. Boys presented significantly higher values ( $p$ $<0,05)$ to $\mathrm{VO}_{2 \max }$ than girls in most stages. Negative, moderate and significant correlation $(p<0,05)$ were obtained between fat mass percentage and $V O_{2 \max }$ for both sexes in all maturation stages. Analysis of the three obesity indicators (BMI, FM percentage $e$ $\Sigma S K F), 11,2 \%$ of the boys and 14,3\% of the girls demonstrated values higher than the normative data.

Conclusions: normal growth standards were verified for both sexes. Children with lower $\mathrm{VO}_{2 \max }$ had a higher percentage of fat mass. Both sexes presented values suggestive of excessive body fat in the beginning of adolescence.
\end{abstract}

Key words Obesity, Body composition, Nutrition assessment, Adolescence

\section{Resumo}

Objetivos: determinar, em escolares portugueses de oito a 16 anos (256 do sexo masculino e 268 do sexo feminino): os padrões de mudanças na composição corporal e no $\mathrm{VO}_{2 m a ́ x}$; a relação entre o percentual de massa gorda (\%MG), índice de massa corporal (IMC) e o somatório de dobras de adiposidade subcutânea ( $\Sigma S K F)$ com o VO2 máx; a percentagem de obesos.

Métodos: o percentual de massa gorda foi estimado do $\mathrm{SSKF}$ (subescapular e tricipital). A amostra foi subdividida em estágios maturacionais de Tanner. A aptidão cardiorrespiratória foi avaliada através do teste Shutlle-run de 20 metros.

Resultados: as variáveis antropométricas e o percentual de massa gorda estiveram de acordo com dados já publicados. Os rapazes apresentaram $\mathrm{VO}_{2 \text { máx }}$ mais elevado $(p<0,05)$ do que as moças na maioria dos estágios. Correlações negativas, moderadas e significativas $(p<0,05)$ entre o percentual de massa gorda o $\mathrm{VO}_{2 \text { máx }}$ foram obtidas em ambos os sexos, em todos os estágios. Na análise dos três indicadores de obesidade (IMC, \%MG e $\Sigma S K F$ ), 11,2\% dos rapazes e 14,3\% das moças apresentaram valores acima dos padrões normativos.

Conclusões: padrões normais de crescimento foram verificados em ambos os sexos. Crianças que apresentaram menor $\mathrm{VO}_{2 \text { máx }}$ também apresentaram maior percentual de massa gorda. Ambos os sexos apresentaram valores sugerindo excesso de gordura corporal no início da adolescência.

Palavras-chave Obesidade, Composição corporal, Avaliação nutricional, Adolescência 


\section{Introdução}

Ao mesmo tempo em que o avanço tecnológico vem contribuindo de forma significativa para elevar o padrão de vida do homem moderno, o aparecimento de muitas doenças crônico-degenerativas que acarretam uma série de riscos para a saúde também vem sendo fruto deste crescimento. 1

$\mathrm{O}$ excesso de peso, a obesidade e a distribuição de gordura corporal têm grande importância enquanto fatores de risco de disfunções cardiovasculares, não apenas pela sua ação deletéria isolada, mas, sobretudo por atuarem como coadjuvantes no desenvolvimento de outros fatores. ${ }^{2}$

O número de crianças e jovens obesos tem aumentado dramaticamente nos países desenvolvidos, constituindo-se atualmente, como um dos tópicos de maior interesse nas análises epidemiológicas da relação entre acumulação de tecido adiposo e o risco para certas doenças, tais como: arteriosclerose, diabetes mellitus, hipertensão e hipercolesterolemia. ${ }^{1}$ Os dados da população portuguesa da cidade do Porto, em Portugal, parecem também sugerir valores de prevalência bastante elevados na população infanto-juvenil. ${ }^{3}$

Contrariamente ao que acontece nos adultos, o começo da obesidade infantil ocorre num contexto complexo e relacionado aos padrões de crescimento físico, fisiológico e psico-social. ${ }^{4} \mathrm{~A}$ idade da manifestação da obesidade parece afetar a probabilidade de sua persistência. Em crianças com início tardio de excesso de peso, a persistência pode aumentar de modo consistente com a canalização da gordura corporal, com a idade e com o crescimento. 5

Neste particular, estão as questões relacionadas às mudanças na distribuição e acumulação do tecido adiposo associadas ao crescimento e à maturação, nomeadamente, a relativa estabilidade dos padrões de distribuição de tecido adiposo nas extremidades e no tronco, este último, representando maior risco para doenças de foro cardiovascular. $6 \mathrm{O}$ entendimento dos padrões de distribuição de gordura em crianças e adolescentes pode fornecer informações importantes sobre as implicações da obesidade para a saúde, além de ser um tópico de interesse para estudos epidemiológicos na análise de distribuição de tecido adiposo e riscos para várias doenças. 4

Nos homens, a quantidade de gordura corporal acima de $20 \%$ e nas mulheres, $30 \%$, caracterizam a obesidade. ${ }^{7}$ Outro método empregado para caracterizar o excesso de peso e a obesidade é a utilização dos percentis 85 e 95, respectivamente, do índice de massa corporal (IMC). É também utilizado o somatório das dobras de adiposidade subcutânea
( $\left.\sum \mathrm{SKF}\right)$, nomeadamente, a tricipital e a subescapular. O IMC e o $\sum$ SKF são os índices mais utilizados por estudos epidemiológicos. Os percentis 85 e 95 para estes dois indicadores são operacionalmente usados para definir obesidade e super-obesidade, respectivamente. Estes valores antropométricos são geralmente referenciados nos dados normativos do National Health and Nutrition Examination Survey I e II (NHANES I e II), ${ }^{8}$ que tem grande representatividade em pesquisas com crianças e adultos e nas tendências da obesidade infantil oferecendo valores específicos dos percentis para idade e sexo. 8

Não obstante os efeitos genéticos e hormonais, o estilo de vida e o contexto social oferecem o cenário perfeito para o desenvolvimento e a evolução da obesidade. A diminuição da atividade física, sendo substituída pela tecnologia moderna, e o aumento do consumo de alimentos com alto teor calórico divulgados pela televisão e outdoors, parecem ser os responsáveis pelo aumento no número de pessoas com excesso de peso. ${ }^{9}$

Em um estudo realizado por Fitzgerald et al. 10 para examinar a relação entre atividade física, tempo dispensado para assistir televisão e índice de massa corporal, foi constatado que crianças que assistem televisão pelo menos três horas/dia têm duas vezes mais probabilidade de serem obesas quando comparadas à aquelas que assistem menos do que uma hora/dia. Uma possível explicação para a associação entre IMC e televisão poderia ser o fato do tempo livre dos indivíduos ser dedicado às atividades sedentárias, ao invés de dedicado a atividades que promovem um aumento do dispêndio energético.

Também o hábito de assistir televisão pode influenciar no tocante à divulgação de alimentos que promovem a obesidade. As pesquisas demonstram que $58 \%$ dos alimentos aconselhados pela televisão durante à tarde contêm altos níveis de gordura e que este veículo de comunicação pode servir como incentivo para o indivíduo alimentar-se de forma não saudável e em grande quantidade. 11

Portanto, a inatividade física parece ser um fator associado com o aumento da prevalência da obesidade. De acordo com relatório sobre atividade física nos Estados Unidos, aproximadamente metade dos jovens entre os 12 e 21 anos não é vigorosamente ativa. 12,13

O reconhecimento das vantagens da prática da atividade física na melhoria da qualidade de vida vem despertando enorme atenção quanto à complexa relação entre os índices de aptidão física e o estado de saúde das pessoas, ${ }^{1}$ particularmente no que respeita aos indicadores metabólicos, como por exemplo, o perfil lipídico. 10 
A função cardiorrespiratória tem sido admitida como a componente de maior relevância da aptidão física relacionada com a saúde. Esta função pode ser avaliada mediante a utilização de diferentes critérios, como por exemplo, a determinação do consumo máximo de oxigênio $\left(\mathrm{VO}_{2 \mathrm{máx}}\right)$ e da capacidade do indivíduo em manter esforços físicos em níveis elevados da sua reserva metabólica. $14 \mathrm{O} \mathrm{VO}_{2 \text { máx }}$ por $\mathrm{kg}$ supostamente o normaliza para as diferenças no tamanho do corpo. Mas a seleção da melhor forma de expressão do $\mathrm{VO}_{2 \text { máx }}$ em crianças é complicada pelo crescimento, e pode ser significativamente influenciada pela maturação biológica e pela composição corporal, especialmente, o conteúdo de gordura.14,15 O nível de treinamento ou de atividade física também podem influenciar os valores do $\mathrm{VO}_{2 \text { máx }}$. Neste sentido, o estudo da relação do $\mathrm{VO}_{2 \text { máx }}$ com os valores de composição corporal considerando o estágio maturacional parece ser relevante.

Assim, este estudo centrou-se nos seguintes objetivos: 1) descrever os valores médios da composição corporal e conhecer os níveis de aptidão cardiorrespiratória de crianças e adolescentes de ambos os sexos divididas segundo o estágio maturacional em que se encontram; 2) relacionar os indicadores de excesso de peso e obesidade com a capacidade cardiorrespiratória através da estimação do consumo máximo de oxigênio $\left(\mathrm{VO}_{2 \text { máx }}\right)$ e do desempenho no Shuttle-run test de 20 metros; 3) verificar a percentagem de crianças e adolescentes que apresentam obesidade de acordo com a avaliação simultânea de três indicadores.

\section{Métodos}

\section{Amostra}

A amostra consistiu de 524 sujeitos (256 do sexo masculino e 268 do sexo feminino), entre oito e 16 anos, aleatoriamente selecionados de 30 escolas da rede pública de ensino na cidade do Porto, Portugal. Estas escolas foram selecionadas de todos os conselhos de forma que pelo menos uma escola representou cada distrito da cidade. Isto permitiu a obtenção de um número proporcional de crianças em função de sua localização geográfica (urbana-central e rural-periférica). As crianças participantes eram selecionadas aleatoriamente do $3^{\circ}$ e $4^{\circ}$ ano do ensino fundamental e $3^{\circ}$ e $4^{\circ}$ ano do ensino médio correspondendo esta seleção aos critérios subjacentes ao sistema de ensino português $\left(1^{\circ}\right.$ e $2^{\circ}$ ciclo).

O Ministério Português para Ciência e Tecnolo- gia concedeu autorização para condução deste estudo. Os pais dos alunos estiveram cientes da realização da pesquisa e, antecipadamente, enviaram um consentimento por escrito permitindo que seus filhos participassem. Para assegurar a discrição dos dados dos sujeitos, estes foram codificados em arquivo de computador. Uma ficha contendo os resultados obtidos pela criança foi enviada aos pais.

\section{Antropometria e composição corporal}

A altura e o peso do corpo foram determinados através de métodos antropométricos padronizados. 16 A altura foi medida entre o vertex e o plano de referência do solo com a criança de pé e encostada ao Holtain Stadiometer portátil. As medidas foram registradas em centímetros, com aproximação à primeira casa decimal (mm). O peso foi medido com aproximação ao kg, com os sujeitos levemente vestidos (camisa e calção) através de uma balança digital portátil SECA 708.

O IMC foi calculado pela razão entre o peso e o quadrado da altura $\left(\mathrm{kg} / \mathrm{m}^{2}\right)$. Foi utilizado um plissômetro Harpenden para obtenção dos valores das dobras de adiposidade subcutânea de dois locais (tríceps e subescapular), de acordo com as técnicas sugeridas por Lukaski. ${ }^{17}$ Cada dobra era medida duas vezes por um técnico treinado e a média aritmética entre os dois valores foi a seguir calculada. Para a obtenção do percentual de massa gorda, isto é, gordura corporal, foram empregadas as equações de predição propostas por Slaughter et al. 18 que utilizam o somatório das dobras de adiposidade subcutânea tricipital e subescapular validada para crianças dos oito aos 18 anos de idade, de acordo com o estágio maturacional.

A distribuição de gordura regional foi obtida pelo cálculo da razão da dobra subescapular e tricipital (S/T). Este valor forneceu um índice dos padrões de distribuição de gordura no tronco. 6

\section{Maturação biológica}

Para verificação do estágio maturacional em que cada criança ou adolescente se encontrava foi elaborada uma ficha de inquérito com figuras do desenvolvimento das mamas, da genitália e da distribuição dos pêlos púbicos segundo os estágios maturacionais de Tanner. 19 Os sujeitos deveriam reconhecer a figura que mais se assemelhava com seu corpo e assinalar em um dos estágios $\left(\mathrm{T}_{1}, \mathrm{~T}_{2}, \mathrm{~T}_{3}, \mathrm{~T}_{4}\right.$ e $\left.\mathrm{T}_{5}\right)$. A maturação é normalmente avaliada utilizando-se um ou mais indicadores de desenvolvimento somático, sexual ou esquelético. ${ }^{20}$ Os estágios de Tanner são 
os mais utilizados para avaliar a maturação sexual, principalmente em estudos transversais, 21 como acontece com o presente estudo.

A auto-avaliação dos sujeitos em relação a sua maturação sexual utilizando os estágios de Tanner demonstrou uma moderada para alta correlação tanto para os rapazes quanto para as moças. Em nosso estudo foi obtido um coeficiente de correlação de Pearson de 0,73 para o teste-reteste de confiabilidade realizado em uma sub-amostra de 50 sujeitos.

\section{Aptidão cardiorrespiratória}

O Shuttle run test de 20 metros proposto por Léger e Lambert, 22 foi utilizado para estimar o $\mathrm{VO}_{2 \text { máx }}$. Os participantes deveriam correr ida-e-volta uma distância de 20 metros. A velocidade inicial é de $8,5 \mathrm{~m} / \mathrm{s}$, aumentando $0,5 \mathrm{~m} / \mathrm{s}$ a cada minuto. Uma gravação fita cassete foi utilizada para orientar o tempo de execução de cada série.

Todas as medições foram realizadas em condições padronizadas. Os resultados foram convertidos para a predição do consumo máximo de oxigênio $\left(\mathrm{VO}_{2 \text { máx }}\right)$, de acordo com as equações de Léger e Lambert.22

Vários estudos têm aplicado o Shuttle-run de 20 metros para estimar o consumo máximo de oxigênio em crianças. 23,24 Este teste foi validado em condições laboratoriais, mostrando uma boa correlação $(r=0,72, p<0,05)$ com o $V_{2 \text { máx }}$ avaliado pelo tapete ergométrico. ${ }^{25} \mathrm{Em}$ nosso estudo, a confiabilidade do teste e re-teste também foi verificada e para ambos os sexos obtivemos uma boa correlação (rapazes $r=0,91$ e moças $r=0,87$ ).

\section{Análise estatística}

A análise das diferenças em cada variável por sexo e estágio maturacional foi efetuada a partir do teste " $\mathrm{t}$ " para medidas independentes. A análise de variância (ANOVA) foi utilizada para testar as diferenças entre os grupos maturacionais do mesmo sexo, seguida pelo teste de Scheffé para comparações múltiplas. O coeficiente de correlação de Pearson foi utilizado para verificar a associação entre o $\mathrm{VO}_{2 \text { máx }}$ e os indicadores de obesidade. O nível de significância para todas as análises foi de $\mathrm{p} \leq 0,05$. Para análise estatística foi utilizado o programa SPSS para Windows.

\section{Resultados}

A Tabela 1 apresenta a estatística descritiva e as significâncias com os valores médios obtidos em cada variável e por estágios maturacionais para ambos os sexos.

Nas variáveis antropométricas peso e altura, observamos que os rapazes obtiveram valores médias inferiores aos das moças em $\mathrm{T}_{3}(\mathrm{p}<0,05)$; entretanto, em $\mathrm{T}_{5}$ estas apresentaram uma diferença para menos $(\mathrm{p}<0,01)$. Relativamente às dobras de adiposidade subcutânea tricipital e subescapular e ao $\sum \mathrm{SKF}$, os rapazes apresentaram valores significantemente inferiores às moças nos estágios maturacionais $\mathrm{T}_{4}$ e $\mathrm{T}_{5}$. Quanto à $\mathrm{S} / \mathrm{T}$, os rapazes apresentaram valores médios superiores em $\mathrm{T}_{2}, \mathrm{~T}_{4}(\mathrm{p}<0,05)$ e $T_{5}(p<0,01)$ quando comparados às moças. Comparando os sexos quanto aos valores médios do $\mathrm{VO}_{2 \text { máx }}$ e ao número de voltas no Shuttle-run test, foi verificado que os rapazes apresentaram valores superiores em $\mathrm{T}_{1}, \mathrm{~T}_{4}$ e $\mathrm{T}_{5}$ ( $\left.\mathrm{p}<0,01 \mathrm{e} \mathrm{p}<0,05\right)$. As moças, entretanto, apresentaram valores médios mais elevados para \%MG nos estágios $\mathrm{T}_{3}(\mathrm{p}<0,05)$, $\mathrm{T}_{4}$ e $\mathrm{T}_{5}(\mathrm{p}<0,01)$ em relação aos rapazes.

$\mathrm{Na}$ análise das diferenças entre os estágios dentro de cada sexo, relativamente aos rapazes, verificamos que $\mathrm{T}_{3}$ apresentou diferença significativa em relação aos demais estágios nas variáveis: peso, altura, razão entre as dobras (S/T) e número de voltas do Shuttle-run test $(\mathrm{p}<0,05)$. Nas moças, essa diferença foi observada predominantemente em $\mathrm{T}_{2}(\mathrm{p}<$ $0,05)$.

A partir do $4^{\circ}$ estágio, as moças demonstraram uma pequena instabilidade nos valores médios de $\mathrm{S} / \mathrm{T}$, com $\mathrm{T}_{1}$ diferindo de $\mathrm{T}_{3}$ e $\mathrm{T}_{5}$.

Quanto ao $\mathrm{VO}_{2 \text { máx }}$ houve um aumento progressivo a partir do $T_{2}$ para os rapazes, mas só foi observada diferença estatística quando este estágio foi comparado a $\mathrm{T}_{5}$. Contrariamente, em $\mathrm{T}_{5}$ as moças apresentaram valores médios do $\mathrm{VO}_{2 \text { máx }}$ menores do que em $\mathrm{T}_{1}, \mathrm{~T}_{2}, \mathrm{~T}_{3}$ e $\mathrm{T}_{4}(\mathrm{p}<0,05)$ 
Valores médios $(\bar{X} \pm d p)$ das variáveis antropométricas, percentagem de massa gorda (\%MG) e VO 2 máx, em ambos os sexos conforme os estágios maturacionais de Tanner

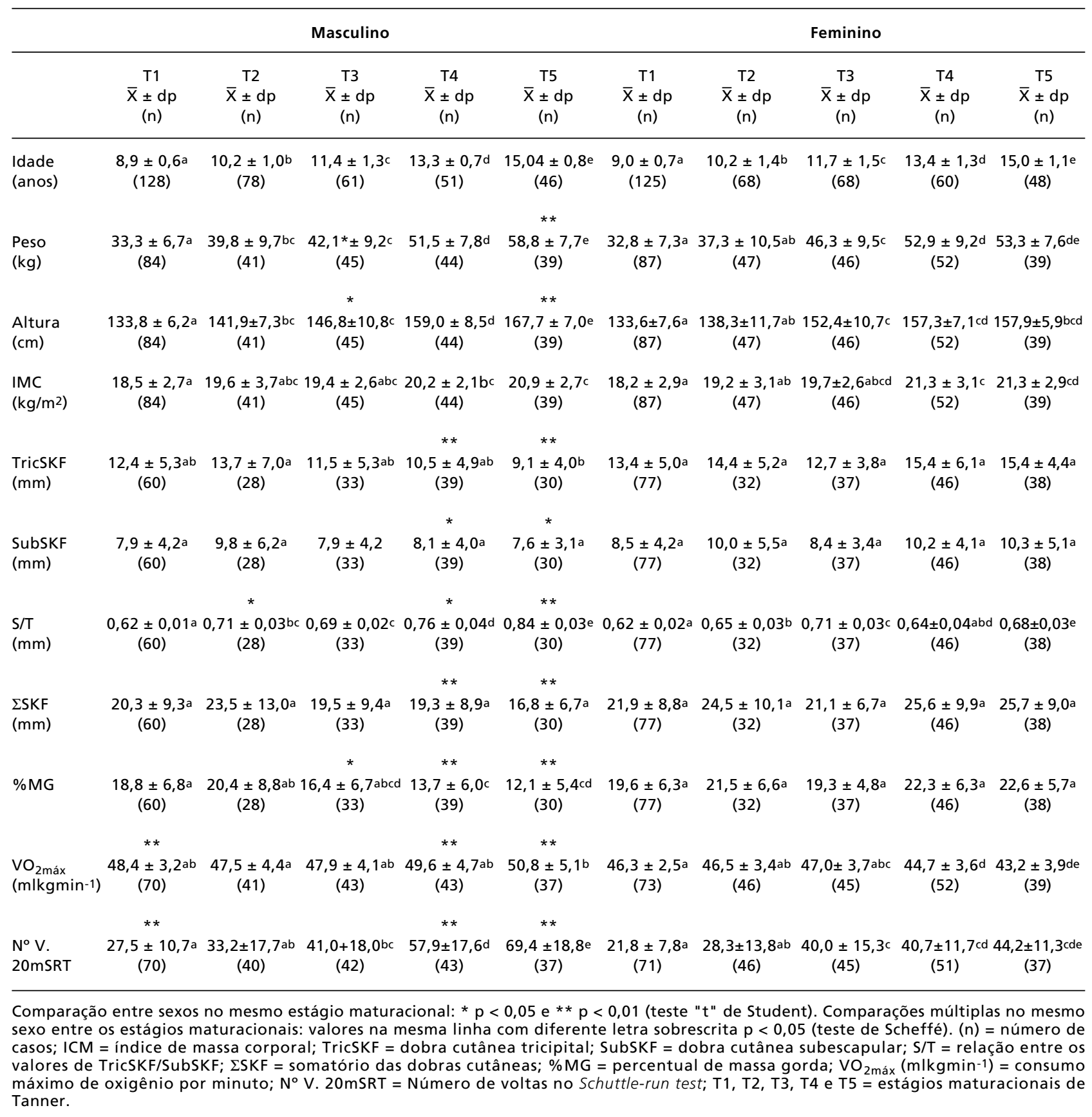


$\mathrm{Na}$ análise das correlações (Tabela 2) obtidas entre os indicadores de obesidade e o $\mathrm{VO}_{2 \text { máx }}$ relativo ao peso do corpo, verificamos correlações negativas, moderadas e significativas ( $p<0,05$ e $p<0,01)$ entre a percentagem de massa gorda bem como o IMC e $\sum \mathrm{SKF}$ com o $\mathrm{VO}_{2 \text { máx }}$ em todos os estágios de maturação, para os rapazes. Para as moças, as correlações foram negativas, moderadas e significativas nos estágios $\mathrm{T}_{2}, \mathrm{~T}_{3}$ e $\mathrm{T}_{5}$ entre os indicadores de obesidade e o $\mathrm{VO}_{2 \text { máx }}$.
$\mathrm{Na}$ análise em simultâneo dos três indicadores de obesidade (IMC, \%MG e $\Sigma$ SKF), $11,2 \%$ dos rapazes e $14,3 \%$ das moças da nossa amostra apresentaram valores acima dos padrões normativos. Destes, a maior percentagem foi observada no estágio $T_{1}$ para $o$ sexo masculino, e no estágio $\mathrm{T}_{2}$ para o sexo feminino.

Tabela 2

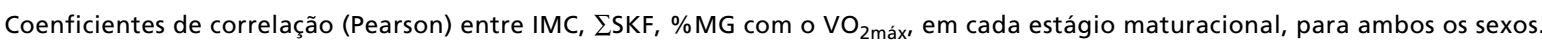

\begin{tabular}{|c|c|c|c|c|c|c|}
\hline \multirow[b]{3}{*}{ Estágios } & \multicolumn{6}{|c|}{$\mathrm{VO}_{2 \text { máx }}$} \\
\hline & \multicolumn{3}{|c|}{ Masculino } & \multicolumn{3}{|c|}{ Feminino } \\
\hline & IMC & $\Sigma S K F$ & $\% \mathrm{MG}$ & IMC & $\Sigma S K F$ & $\%$ MG \\
\hline $\mathrm{T} 1$ & $-0,39 * *$ & $-0,36$ ** & $-0,34^{*}$ & $-0,19$ & $-0,27$ * & $-0,26^{*}$ \\
\hline $\mathrm{T} 2$ & $-0,45 * *$ & $-0,52 * *$ & $-0,42^{*}$ & $-0,33^{*}$ & $-0,49 * *$ & $-0,49 * *$ \\
\hline T3 & $-0,36$ * & $-0,50 * *$ & $-0,50$ ** & $-0,36^{*}$ & 0,37 * & $-0,37$ * \\
\hline $\mathrm{T} 4$ & $-0,40$ ** & $-0,68 * *$ & $-0,70 * *$ & 0,05 & $-0,14$ & $-0,13$ \\
\hline T5 & $-0,29$ & $-0,41$ * & $-0,40 *$ & $-0,57 * *$ & $-0,53 * *$ & $-0,57 * *$ \\
\hline
\end{tabular}

* $\mathrm{p}<0,05$, ** $\mathrm{p}<0,01, \mathrm{ICM}=$ índice de massa corporal, $\Sigma \mathrm{SKF}=$ somatório das dobras cutâneas, \% MG = percentual de massa gorda $\mathrm{VO}_{2 \text { máx }}=$ consumo máximo de oxigênio.

\section{Discussão}

Relativamente às variáveis antropométricas, a nossa amostra quando comparada a outras apresentou uma evolução gradual semelhante em estudos no mesmo domínio.19,21,26,27 A curva de crescimento da infância até a adolescência não é muito diferente de uma criança para a outra, mas existem consideráveis variações interindividuais no tempo destas mudanças, bem como, nos valores para a altura e o peso entre diferentes crianças da mesma idade cronológica. 26 No presente estudo, observamos que os rapazes apresentaram valores inferiores para o peso no estágio $\mathrm{T}_{3}$ em comparação com as moças. Relativamente à altura, apesar das moças terem obtido valor médio superior aos rapazes em $T_{3}$, essa situação foi similar em $\mathrm{T}_{4}$. De fato, já está bem estabelecido que os valores do peso e altura são similares entre os sexos durante a infância $\left(T_{1}\right.$ e $\left.T_{2}\right), 19,27$ mas os rapazes tendem a ser mais fortes e mais altos no período póspúbere, ${ }^{21,27}$ provavelmente porque têm um período mais longo de crescimento e a proporção do seu crescimento em relação à velocidade máxima durante a puberdade é maior $(9,2$ vezes ou $8,3 \mathrm{~cm}$ no ano máximo).14 Não obstante o peso do indivíduo estar susceptível aos fatores relacionados aos hábitos alimentares e ao estilo de vida, os mecanismos endócrinos, os fatores genéticos e a influência nutricional também estão envolvidos no ganho ponderal. 14,19

Relativamente à tendência de distribuição de gordura, as moças no presente estudo apresentaram uma certa instabilidade ao longo dos estágios maturacionais, enquanto que os rapazes apresentaram um aumento. Estes fatos concordam com os dados obtidos noutros estudos 4,6 que reviram as tendências na distribuição de gordura subcutânea no decorrer do crescimento. Desta forma, em estudos longitudinais que acompanharam crianças dos oito aos 18 anos de idade, se observou que em rapazes no começo da puberdade, existe um aumento da gordura subcutânea localizada na parte central do corpo (abdômen e 
parte superior do tronco) e uma diminuição nas extremidades (tríceps e geminal). Enquanto que nas moças existe um aumento da gordura subcutânea tanto na parte central quanto nas extremidades. ${ }^{29}$ Isto se deve, provavelmente, à ação dos hormônios sexuais que atuam na deposição regional de gordura, nomeadamente, a ação do cortisol e insulina (na lipogênese) e do hormônio do crescimento e esteróides sexuais (na lipólise). 28 Por outro lado, a distribuição de gordura também pode ser influenciada por fatores modificáveis (dieta, massa gorda e nível de atividade física) e não-modificáveis (genes, maturação, sexo e etnia).12,13

Para além da atuação da concentração hormonal, a diferença anatômica no número e densidade de receptores hormonais específicos, na capilarização e na atividade de enzimas que promovem a degradação de lipídeos, podem justificar as diferenças entre os sexos. 29

No que respeita à aptidão cardiorrespiratória, existem numerosos fatores que podem afetar o $\mathrm{VO}_{2 \text { máx }}$, tais como: a idade, o sexo, a quantidade de gordura corporal, a genética, a etnia, a maturação e o nível de treinamento. $20 \mathrm{~A}$ hereditariedade pode ser o principal fator nas variações dos valores do $\mathrm{VO}_{2 \text { máx }}$ entre indivíduos. 14,30 No nosso estudo, os rapazes comparados às moças apresentaram valores superiores em $\mathrm{T}_{1}, \mathrm{~T}_{4} \mathrm{eT}_{5}$. Na fase puberal, as moças atingiram uma relativa estabilidade quanto ao $\mathrm{VO}_{2 \text { máx }}$, enquanto que os rapazes continuaram a aumentar; 14 este mesmo perfil foi encontrado por Janz et al. 20 Mesmo com as tentativas de normalizar o consumo máximo de $\mathrm{O}_{2}$ para o peso do corpo, ainda não está clara a relação entre o $\mathrm{VO}_{2 \text { máx }}$ e as modificações que ocorrem no tamanho do corpo de uma criança em crescimento. Durante a infância o peso corporal triplica, a quantidade de gordura nos rapazes está em cerca de $15 \%$ do peso do corpo, mas nas moças da mesma idade a percentagem de gordura pode chegar a representar $23 \%$ do peso total do corpo. 15 Ademais, os rapazes apresentam, geralmente, maior estatura, maior massa muscular no ventrículo esquerdo, e são fisicamente mais ativos do que as moças que, por sua vez, apresentam maior quantidade de gordura corporal.14 Também existem dúvidas se a aptidão aeróbica aumenta por causa do aumento do tamanho do corpo ou por causa da melhoria na capacidade funcional que é independente das dimensões corporais, ou ainda por ambos.1,14,15

O Shuttle-run test de 20 metros pode ser também um indicador de aptidão cardiorrespiratória. 16 Foram observadas diferenças $\left(\mathrm{T}_{1}, \mathrm{~T}_{4} \mathrm{eT}_{5}\right)$ entre os sexos nos valores médios do número de voltas que podem estar relacionadas ao aumento da massa muscular e diminuição da massa gorda verificadas nos adolescentes masculinos, os quais podem afetar o tecido metabolicamente ativo, influenciando diretamente no $\mathrm{VO} 2_{\text {máx }}{ }^{2}$ Por outro lado, a atividade física regular pode também estar diretamente associada ao melhor desempenho dos rapazes em relação às moças. 21 Nas atividades que requerem movimento do peso corporal, as moças são geralmente penalizadas por carregarem excesso de massa gorda e é provável que os rapazes apresentem para os valores da razão de $\mathrm{VO}_{2 \text { máx }}$ por kg de massa magra, $10 \%$ a mais do que as moças. 15

Os valores do $\mathrm{VO}_{2 \text { máx }}$ das crianças e adolescentes do nosso estudo apresentaram correlações negativas na sua generalidade com os três indicadores de obesidade (IMC, $\Sigma$ SKF e \%MG). Dessa forma, a quantidade de gordura corporal pode afetar os valores do $\mathrm{VO}_{2 \text { máx }}$, quando este é expresso em termos relativos ao peso corporal. A obesidade, por sua vez, parece não afetar a aptidão cardíaca, mas certamente limitará o desempenho em testes de resistência e a um menor valor do $\mathrm{VO}_{2 \text { máx }} \cdot{ }^{14}$ Estes resultados também foram encontrados em estudos com crianças de ambos os sexos realizados por Mueller et al. 27

A utilização do IMC para avaliar o grau de obesidade deve ser considerada com cuidado, pois valores elevados podem decorrer dos atributos físicos adquiridos pelos indivíduos, principalmente durante o crescimento. 26 Os cálculos da percentagem de massa gorda podem também superestimar a prevalência da obesidade, pois as equações de predição que utilizam o $\sum$ SKF podem não considerar as mudanças inerentes ao processo maturacional, 18 porém uma análise simultânea destes indicadores minimizaria os possíveis erros de interpretação.

O fato de este estudo ter sido transversal pode ter comprometido a consistência das comparações. Um estudo longitudinal seria o ideal quando levamos em consideração as mudanças que ocorrem durante o crescimento.

\section{Agradecimentos}

Fundação para Ciência e Tecnologia pelo financiamento do Projeto de Investigação através do Programa PRAXIS XXI: PSAU/C/SAL/122/96 


\section{Referências}

1. Sallis J, Owen N. Physical activity and behavioural \& medicine: behavioural medicine and health psychology. New York: Sage; 1999. v. 3.

2. Bouchard C. The obesity epidemic: introduction. In Bouchard C, editor. Physical activity and obesity. Champaign, IL: Human Kinetics; 2000. p. 3-20.

3. Mota J, Ribeiro JC, Guerra S, Leandro C, Duarte J. Valores de referência da obesidade em crianças e adolescentes na área do grande Porto. Endocrinol Met Nutr 2000: 5: 24151

4. Lohman TG. Advances in body composition assessment. Champaign, IL: Human Kinetics; 1992.

5. Armstrong N, Welsman J. Young people and physical activity. New York: Oxford University; 1997.

6. Malina RM, Koziel S, Bielicki T. Variation in subcutaneous adipose tissue distribution associated with age, sex, and maturation. Am J Hum Biol 1999; 11: 189-200.

7. Bray GA. An approach to the classification and evolution of obesity. In: Bjorntorp P, Brodoff BN, editors. Obesity. New York: Lippincott; 1992. p.294-308.

8. Himes JH, Dietz WH. Guidelines for overweight in adolescent preventive services: recommendations from an expert committee. Am J Clin Nutr 1994; 59: 307-16.

9. Taylor WC, Blair SN, Cumimgs SS, Wun CC, Malina RM. Childhood and adolescence physical activity patterns and adults physical activity. Med Sci Sports Exerc 1999; 31: $118-23$

10. Fitzgerald SJ, Kriska AM, Pereira MA, DeCourten MP. Associations among physical activity, television watching, and obesity in adult pima Indians. Med Sci Sports Exerc 1997; 29: 910-5.

11. NIH. National Institute of Health. Clinical guidelines on the identification, evaluation, and treatment of overweight and obesity in adults. Bethesda: National Institute of Health, National Heart Lung, Blood Institute; 1988.

12. Raitakari OT, Taimela S, Porkka KVK, Telama R, Valimaki I, Akerblom HK, Vaikari JSA. Association between physical activity and risk factors for coronary heart disease: the cardiovascular risk in young Finns study. Med Sci Sports Exerc 1997; 29: 1055-61.

13. Ebbeling S, Rodrigues P. Effects of exercise combined with diet therapy on protein utilization in obese children. Med Sci Sports Exerc 1999; 31: 378-85.

14. Rowland TW. Developmental exercise physiology. Champaign, IL.: Human Kinetics; 1996.

15. Malina RM, Bouchard C. Subcutaneous distribution during growth. In: Bouchard C, Johnston FE, editors. Fat distribution during growth and later health outcomes. New York: Alan R. Liss; 1988. p. 63-84.
16. Council of Europe. Eurofit handbook for the eurofit tests of physical fitness. Rome: Italian National Olympic Committee; 1988.

17. Lukaski HC. Methods for the assessment of human body composition: traditional and new. Am J Clin Nutr 1987; 46: $537-56$

18. Slaughter MH, Lohman TG, Boileau RA, Horswill CA, Stillman RJ, Van Loan MD, Bembem DA. Skinfold equation for estimation of body fatness in children and youth. Hum Biol 1988; 60: 709-23.

19. Marshall WA, Tanner JM. Puberty. In: Falkner F, Tanner JM, editors. Human growth. New York: Plenun; 1986. v. 2, p.171-210.

20. Janz KF, Burns TL, Witt JD, Mahoney LT. Longitudinal analysis of scaling $\mathrm{VO}_{2}$ for differences in body size during puberty: the muscatine study. Med Sci Sports Exerc 1998; 8: 1436-44

21. Hitchen PJ, Jones MA, Stratton G. Maturity and gender effects on selected physical fitness parameters in high school age children. J Sports Sci 1999; 17: 18-9.

22. Léger A, Lambert J. A maximal multistage $20 \mathrm{~m}$ Shuttle run test to predict $\mathrm{VO}_{2 \text { máx }}$. Eur J Appl Physiol 1982; 49: 112 .

23. Ahmaidi S, Collomp K, Cailaud C, Prefaut C. The effect of Shuttle-run test protocol and the resulting lactacidaemia on maximal velocity and maximal oxygen uptake during the shuttle exercise test. Eur J Appl Physiol 1992; 65: 475-9.

24. Van Mechelen W, Hlobil H, Kemper HCG. Validation of two running tests as estimates of maximal aerobic power in children. Eur J Appl Physiol 1986; 55: 503-6.

25. Barnett GP, Chan LYS, Bruce A. Preliminary study of the 20-m multistage shuttle-run as a predictor of peak of $\mathrm{VO}_{2}$ in Hong Kong Chinese students. Paediatr Exerc Sci 1993; 5: 42-50.

26. Malina RM, Bouchard C. Growth, maturation and physical activity. Champaingn, IL: Human Kinetics; 1991.

27. Mueller WH, Harrist RB, Doyle RB, Ayars CL, Labarthe DR. Body measurement variability, fatness, and fat-free mass in children 8,11 , and 14 years of age: project heart beat! Am J Hum Biol 1999; 11: 69-78.

28. Bjorntorp P. Regional obesity. In: Bjorntorp P, Brodoff BN editors. Obesity. New York: Lippincott; 1992. p. 579-86.

29. Roemmich JN, Rogol AD. Hormonal changes during puberty and their relation to fat distribution. Am J Hum Biol 1999; 11: 209-22

30. Sharkey BJ. Physiology and fitness. 3rd ed. Champaigne, IL: Human Kinetics Books; 1990. 